\title{
ORTHOGONALITY GRAPH OF THE ALGEBRA OF UPPER TRIANGULAR MATRICES
}

\author{
B. R. BAKHADLY
}

Abstract. We study the connectedness and the diameter of orthogonality graphs of upper triangular matrix algebras over arbitrary fields.

Mathematics subject classification (2010): 15A24, 05C50.

Keywords and phrases: Orthogonality, graphs, upper triangular, matrices.

\section{REFERENCES}

[1] S. Akbari, M. Ghandehari, M. Hadian, A. Mohammadian, On commuting graphs of semisimple rings, Linear Algebra Appl. 390 (2004), 345-355.

[2] S. AkBARI, A. Mohammadian, On the zero-divisor graph of a commutative ring, J. Algebra 274 (2004), 847-855.

[3] S. Akbari, A. Mohammadian, Zero-divisor graphs of non-commutative rings, J. Algebra 296 (2006), 462-479.

[4] S. Akbari, A. Mohammadian, H. Radjavi, P. Raja, On the diameters of commuting graphs, Linear Algebra Appl. 418 (2006), 161-176.

[5] S. AKBARI, P. RAJA, Commuting graphs of some subsets in simple rings, Linear Algebra Appl. 416 (2006), 1038-1047.

[6] B. R. Bakhadly, A. E. Guterman, O. V. Markova, Graphs defined by orthogonality, Computational methods and algorithms. Part XXVII, Zap. Nauchn. Sem. POMI 428 (2014), 49-80 (in Russian). Journal of Mathematical Sciences (New York) 207: 5 (2015), 698-717 (in English).

[7] J. K. BAKSALARY, J. HAUKE, A further algebraic version of Cochran's theorem and matrix partial orderings, Linear Algebra Appl. 127 (1990), 157-169.

[8] T. Fenstermacher, E. Gegner, Zero-Divisor Graphs of $2 \times 2$ Upper Triangular Matrix Rings Over $\mathbb{Z}_{n}$, Missouri Journal of Math. Sciences 26: 2 (2014), 151-167.

[9] A. E. Guterman, M. A. Efimov, Monotone maps on matrices of index one, Zap. Nauchn. Sem. POMI 405 (2012), 67-96 (in Russian). Journal of Mathematical Sciences (New York) 191: 1 (2013), 36-51 (in English).

[10] F. HARARY, Graph Theory, Addison Wesley, 1969.

[11] A. LI, R. P. TuCCI, Zero Divisor Graphs of Upper Triangular Matrix Rings, Communications in Algebra 41: 12 (2013), 4622-4636.

[12] B. LI, Zero-Divisor Graph of Triangular Matrix Rings over Commutative Rings, International Journal of Algebra 5: 6 (2011), 255-260.

[13] P. G. Ovchinnikov, Automorphisms of the poset of skew projections, J. of Functional Analysis 115 (1993), 184-189.

[14] P. ŠEMRL, Order-preserving maps on the poset of idempotent matrices, Acta Sci. Math. (Szeged) 69 (2003), 481-490. 